\title{
Hydride Growth Mechanism in Zircaloy-4: Investigation of the Partitioning of Alloying Elements
}

Isabelle Mouton $^{1 *}$, Yanhong Chang ${ }^{1}$, Siyang Wang ${ }^{2}$, Andrew J. Breen ${ }^{1}$, Agnieszka Szczepaniak ${ }^{1}$, Leigh T. Stephenson ${ }^{1}$, Dierk Raabe ${ }^{1}$, T. Ben Britton ${ }^{2}$ and Baptiste Gault ${ }^{1}$

1. Max-Planck-Institut für Eisenforschung, Düsseldorf, Germany.

2. Department of Materials, Royal School of Mines, Imperial College London, London, UK.

* Corresponding author: isabelle.mouton42@gmail.com

Zirconium-based alloys are typically used for nuclear fuel cladding due to their low thermal neutron capture, high temperature corrosion resistance and relatively good mechanical properties. Among these alloys, the most common is Zircaloy-4 that has a base composition of of $\mathrm{Zr}-1.5 \% \mathrm{Sn} \_0.2 \% \mathrm{Fe}-0.1 \% \mathrm{Cr}$ (wt\%). It has previously been shown that $\mathrm{Fe}$ and $\mathrm{Cr}$ atoms are often observed as secondary-phase particles of the Laves phase or segregation at grain boundaries (GBs) [1]. A recent study also highlights the presence of Sn at the growth front of GB hydride [2]. Nevertheless, investigation of the mechanism of GB hydride growth are still missing. The chemical sensitivity and high spatial resolution of atom probe tomography (APT) makes the technique well suited to detect segregation of alloying additions to interfaces for example. Electron backscattered diffraction (EBSD) enables targeted preparation of GBs with known misorientation angle which can then be correlated with their chemistry.

Here, we firstly investigate quantitatively the distribution of alloying element at GBs in the as-received, the annealed $\left(800^{\circ} \mathrm{C}, 336 \mathrm{~h}\right)$ as well as hydrogen-charged Zircaloy-4 using EBSD and APT. Figure 1, shows the EBSD map, APT reconstruction and the composition profiles of the main alloying elements obtained perpendicularly to the GB. Figure $1 \mathrm{a}$ and $\mathrm{b}$ show the evolution of Fe and Sn content at GBs between two $\alpha$-Zr grains (in purple in Figurela and b) in the as-received fine grain and in the annealed 'blocky alpha' sample with large grains before hydrogen charging [3]. As observed in the EBSD map, even if the grain size changes drastically, both GBs contain Fe and Sn enrichment with similar composition ( $\sim 2 \%$ at. Fe \& $\sim 1.5 \%$ at. Sn). After hydrogen charging, a large number of hydrides were found at GBs, as reported by Birch, et al [4]. APT specimens were extracted at a GB where intergranular hydrides had formed on both sides (in yellow in the Figure1c). The corresponding composition profile shows that the hydrogen content is lower at the GB which only contains approx. $0.3 \%$ at. Fe and approx. $0.3 \%$ at. Sn. Therefore, GB chemistry in Zircaloy-4, enriched in $\mathrm{Sn}$ and $\mathrm{Fe}$, is also stable during annealing and/or hydrogen charging. Secondly, to explore the hydride growth mechanism in Zircaloy-4, the atomic scale environment of the hydride growth front is investigated. APT reconstruction in Figure 2 shows two distinct phases: a hydride phase in yellow and $\alpha-\mathrm{Zr}$ in purple, additionally the Sn distribution is highlighted by a green iso-surface. Segregation of $\mathrm{Sn}$ is observed along the hydride growth front (as previously observed in [2]) but Sn also exhibits an inhomogeneous distribution within the Zr-alpha phase ahead of the hydride growth front. The planar features are likely stacking faults decorated by Sn.

Both observations enable significantly better understanding of the hydride growth mechanism in Zircaloy-4. The proposed mechanism is that hydrides grow mainly from Fe rich GBs without changing the local chemical nature. During the hydride growth, Sn atoms diffuse out of the hydride phase, and segregate to the growth front. Stacking faults form likely due to the volume expansion associated to hydride growth through gliding of $<10-10\rangle$ partial dislocations on alternate basal planes as proposed by Carpenter [5] and Sn gets trapped at these defective features. 


\section{References:}

[1] Y Dong, AT Motta and EA Marquis, Journal of Nuclear Materials 442 (2013), p. 270.

[2] AJ Breen et al., Scripta Materialia 156 (2018), p. 42.

[3] VS Tong and TB Britton, Acta Materialia 129 (2017), p. 510.

[4] R Birch et al., arXiv:1807.11927 [cond-mat] (2018).

[5] GJC Carpenter, Acta Metall. 26 (1978), p. 1225.

(a) As-received Zircaloy-4
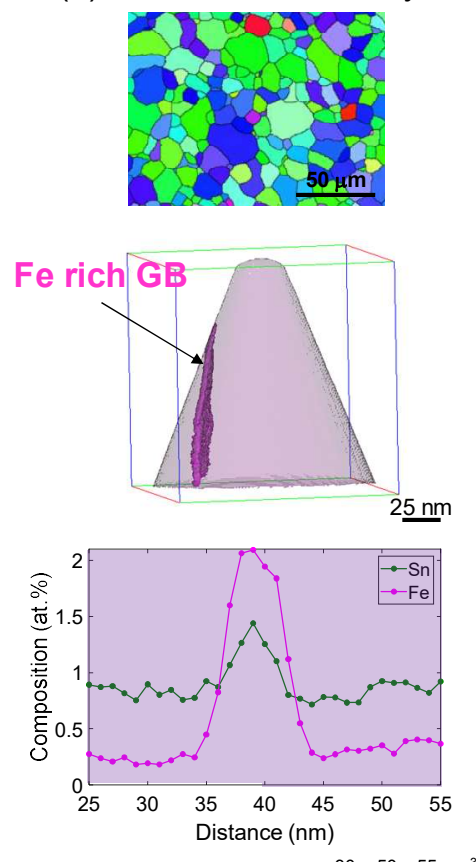

(b) Annealed Zircaloy-4
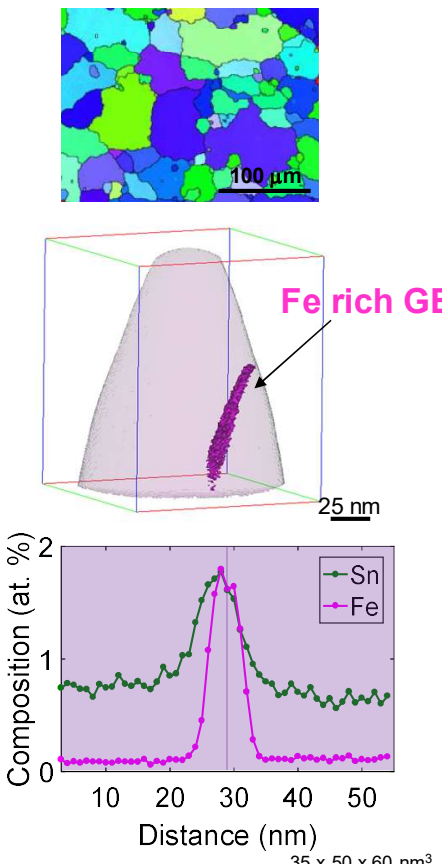

(c) H charged Zircaloy-4
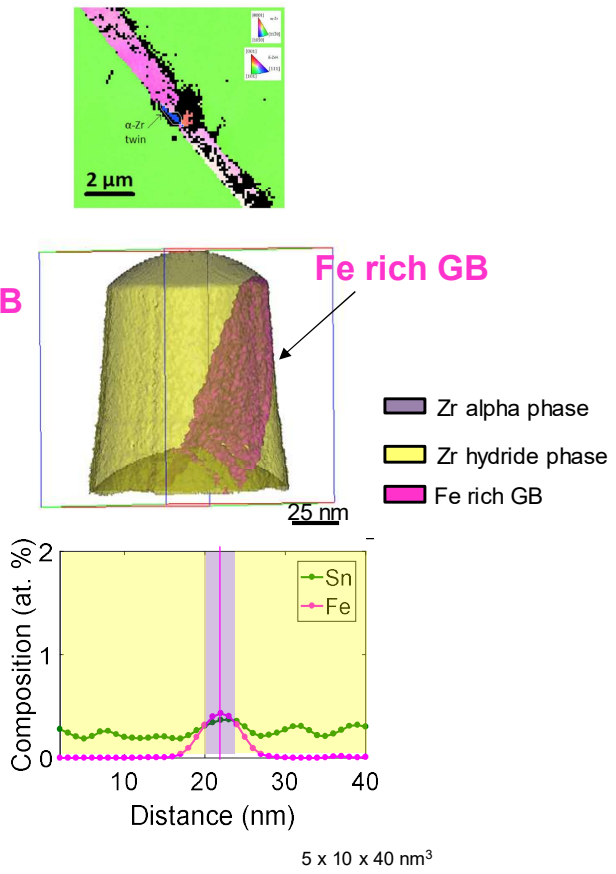

Figure 1. EBSD maps and APT reconstruction containing Fe-rich GB in (a) as-received fine grain, (b) annealed 'blocky alpha' large grain and (c) hydrogen charged Zircaloy-4 and their corresponding chemical composition profiles.

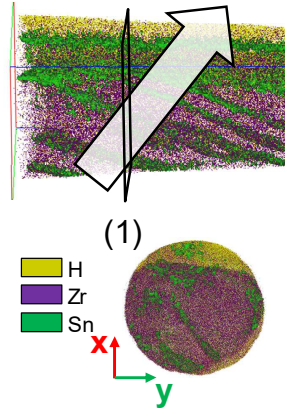

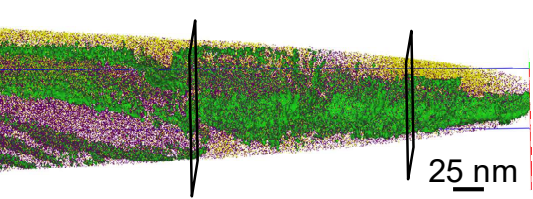

(2)

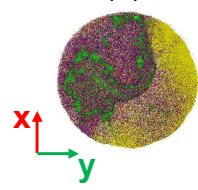

(3)

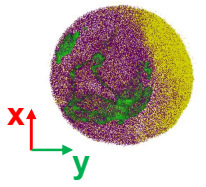

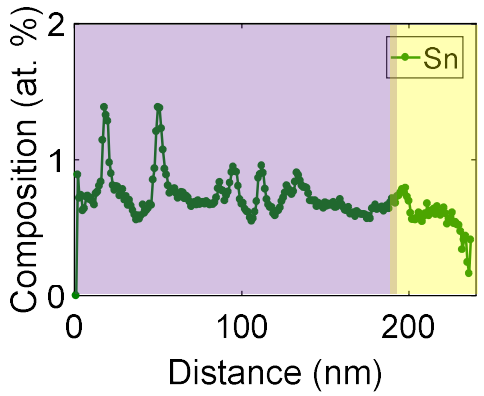

Figure 2. APT reconstruction of hydrogen-charged Zircaloy-4 showing the chemical environment of the hydride growth front. 